DAMTP-2007-67

\title{
Dynamics in Nonlocal Cosmological Models Derived from String Field Theory
}

\author{
Liudmila Joukovskaya* \\ DAMTP, Centre for Mathematical Sciences, University of Cambridge, \\ Wilberforce Road, Cambridge CB3 0WA, UK
}

\begin{abstract}
A general class of nonlocal cosmological models is considered. A new method for solving nonlocal Friedmann equations is proposed, and solutions of the Friedmann equations with nonlocal operator are presented. The cosmological properties of these solutions are discussed. Especially indicated is $p$-adic cosmological model in which we have obtained nonsingular bouncing solution and string field theory tachyon model in which we have obtained full solution of nonlocal Friedmann equations with $w=-1$ at large times. The possibility of obtaining realistic value of cosmological constant from nonlocal cosmological models is also discussed.
\end{abstract}

\section{Introduction}

In recent works there appears interest in nonlocal cosmological models derived from String Field Theory in connection with problem of describing cosmological inflation or accelerating expansion of the Universe.

Modern cosmological data indicates that expansion of the Universe is accelerating. It may be owing to a component of the Universe with negative pressure, Dark Energy. Recent results of WMAP [1] together with Ia supernovae data give us the following range for the dark energy state parameter $w=-0.97_{-0.09}^{+0.07}$. In the assumption that the value of the state parameter changes in the range $0>w>-1$ there exist different theoretical models for the dark energy (see reviews [2,3] and references therein) - quintessence models [4], K-essence [5], quintom models [6], models constructed on the DBI action (see reviews [7] and references therein, [8]) and dilatonic models [9]. The case $w=-1$ corresponds to models with cosmological constant, which are also possible. We also can find in the literature models related $w<-1$ with modified gravity [10].

A nontrivial possibility is the case $w<-1$, that means the violation of the null energy condition (NEC). One of the possibilities is to consider the phantom Universe

*E-mail: 1.joukovskaya@damtp.cam.ac.uk 
$[11,12]$. There were proposed several coupled scalar-gravity models in which the null energy condition is violated, but as an effect most such models are unstable [13]. Recently it was proposed another possibility to consider dark energy description in the context of $D$-brane decay in cubic superstring field theory [11]. It was shown that $D$-brane decay can be interpreted at least at late times as a phantom model. Note that unlike phenomenological phantom models here phantom is an effective theory. Since string field theory by itself is a consistent quantizable theory this approach does not suffer from instabilities which are inevitable for phenomenological phantom models [11]. Such a nonlocal cosmological models were also considered in [14-18].

At the same time there have been a number of attempts to realize description of the early Universe via nonlocal cosmological models [18-21]. One example is $p$-adic inflation model [19] which is represented by nonlocal $p$-adic string theory coupled to gravity. For this model, a rolling inflationary solution was constructed and the interesting features were discussed and compared with cosmic microwave background (CMB) observations. The possibility of obtaining large nongaussian signatures in the CMB has also been considered in a general class of single field nonlocal hill-top inflation models [20]. Another example is investigation of the inflation near a maximum of the nonlocal potential when non-local derivative operators are included in the inflaton Lagrangian. It was found that higher-order derivative operators in the inflaton Lagrangian can support a prolonged phase of slow-roll inflation near a maximum of the potential [21].

It may happen that both early stage and contemporarily Universe can be explained with a single nonlocal model derived from string field theory. To be able to tell more about the early stage we need to consider perturbations in nonlocal models. It will be very interesting to compare obtained results in particular with those in [22-24]. To be able to do it the new technique for solving nonlinear Friedmann equation is required which will hopefully be provided in the present work where for the first time construction of solution of the full nonlocal Friedmann equations is presented. That is why in the present work we discuss numerical solution of Friedmann equations and their features in more detail.

One of the popular examples of nonlocal models is the $D$-brane decay in the gravitational background in the framework of string field theory which is described by the following tachyon action

$$
S=\int d^{4} x \sqrt{-g}\left(\frac{m_{p}^{2}}{2} R+\frac{\xi^{2}}{2} \phi \square_{g} \phi+\frac{1}{2} \phi^{2}-\frac{1}{4} \Phi^{4}-T-\Lambda^{\prime}\right),
$$

where $g$ is the metric, $m_{p}$ is reduced Planck mass, $\phi$ is tachyon field, $\Phi=e^{k \square} \phi, k=\frac{1}{8}$, $\square_{g}$ is usual Beltrami-Laplace operator, $T$ is tension of the $D 3$ brane, $\Lambda^{\prime}$ is the effective cosmological constant.

Note that covariant string field theory as of today is constructed only in the flat background. It was built only for some special types of non-flat backgrounds, in particular, in the anti-de sitter background [25] and in the plane wave background [26]. So the action (1) at the special choice of $V(\Phi)$ is a direct generalization for the approximated tachyon action to the case of arbitrary metric. 
The action (1) in the Friedmann-Robertson-Walker (FRW) background

$$
d s^{2}=-d t^{2}+a^{2}(t)\left(d x_{1}^{2}+d x_{2}^{2}+d x_{3}^{2}\right)
$$

leads to the following Friedmann equations

$$
\begin{gathered}
3 H^{2}=\frac{1}{m_{p}^{2}} \mathcal{E}, \\
3 H^{2}+2 \dot{H}=-\frac{1}{m_{p}^{2}} \mathcal{P},
\end{gathered}
$$

which have the same form as the usual equations except for extra term in the expression for the energy $\mathcal{E}$ and pressure $\mathcal{P}$ which appear from the nonlocal interaction and which contain the Hubble function. This term makes these Friedmann equations more complicated even for numerical consideration. More precisely,

$$
\begin{aligned}
\mathcal{E} & =\underbrace{\mathcal{E}_{k}+\mathcal{E}_{p}+T+\Lambda^{\prime}}_{\text {usual local energy expression }}+\mathcal{E}_{n l 1}+\mathcal{E}_{n l 2}, \\
\mathcal{P} & =\underbrace{\mathcal{E}_{k}-\mathcal{E}_{p}-T-\Lambda^{\prime}}_{\text {usual local pressure expression }}-\mathcal{E}_{n l 1}+\mathcal{E}_{n l 2},
\end{aligned}
$$

where

$$
\mathcal{E}_{n l 1}=k \int_{0}^{1} d \rho\left(e^{k \rho \square_{g}} \Phi^{3}\right)\left(-\square_{g} e^{-k \rho \square_{g}} \Phi\right), \quad \mathcal{E}_{n l 2}=-k \int_{0}^{1} d \rho\left(\partial e^{k \rho \square_{g}} \Phi^{3}\right)\left(\partial e^{-k \rho \square_{g}} \Phi\right) .
$$

The field equation reads

$$
\left(\xi^{2} \square_{g}+1\right) e^{-\square_{g}} \Phi=V^{\prime}(\Phi),
$$

here and below we will denote by $/$ usual function derivative. The Beltrami-Laplace operator takes the form $\square_{g}=-\partial_{t}^{2}-3 H(t) \partial_{t}+\frac{1}{a^{2}} \partial_{x}^{2}$.

In the case of cubic potential the action (1) corresponds to the the tachyon in bosonic String Field Theory [27] for the lowest level in the level truncated scheme [28,29] with the induced twisted space-time. It is supposed that that we are dealing with $D 3$-brane in the D26 space-time and the volume of the D22 compactified subspace is omitted.

The case of quartic potential in the action (11) corresponds to inclusion of the metric in the tachyon action of the fermionic cubic string [30] in the approximation of a slowly varying auxiliary field [31]. Such an action appears in the level truncated scheme where only lowest scalar field (tachyon field) in the $\mathrm{GSO}(-)$ sector and correspondingly the lowest scalar field in the $\mathrm{GSO}(+)$ sector (this field does not have kinetic term and is considered as auxiliary field) are considered. Integration over the auxiliary field results in the quartic potential for the tachyon field. Note that appearance of the non-extremal $D$-brane in the framework of fermionic string field theory requires $\operatorname{GSO}(-)$ sector in the spectrum $[32,33]$.

The purpose of this work is to consider a general class of nonlocal cosmological models of the form (11) and discuss which physical properties and consequences might be obtained 
from such models. Among them we will be interested firstly in the classical solutions of the corresponding Friedmann equations which can be considered as a first approximation to the quantum solutions and might be useful for the study of ways to avoid the cosmological singularity problem [34].

We start with the description of some general class of nonlocal models. In section 3 we will describe new numerical algorithm for solving nonlocal Friedmann equations. In section 4 we will present the numerical solutions of Friedmann equations for different parameters and will provide analytical explanation for some of their interesting features. In section 5 we will consider which cosmological solutions can be provided by nonlocal models.

\section{General Class of Nonlocal Cosmological Models}

In this paper we will consider the following nonlocal scalar field on the $D$-brane coupled to the gravity

$$
S=\int d^{4} x \sqrt{-g}\left[\frac{M_{p}^{2}}{2} R+\frac{M_{s}^{4}}{g_{4}}\left(\frac{\xi^{2}}{2} \phi\left(\square_{g} / M_{s}^{2}\right) \phi+\frac{1}{2} \phi^{2}-V\left(e^{k \square_{g} / M_{s}^{2}} \phi\right)-\Lambda^{\prime}-T\right)\right],
$$

where $g$ is the metric, $\square_{g}=\frac{1}{\sqrt{-g}} \partial_{\mu} \sqrt{-g} g^{\mu \nu} \partial_{\nu}, M_{p}$ is a Planck mass, $M_{s}$ is a characteristic string scale related with the string tension $\alpha^{\prime}$ as $M_{s}=1 / \sqrt{\alpha^{\prime}}, \phi$ is a scalar field, $g_{4}$ is a dimensionless four dimensional effective coupling constant related with the ten dimensional string coupling constant $g_{0}$ and the compactification scale, $\Lambda=\frac{M_{s}^{4}}{g_{4}} \Lambda^{\prime}$ is an effective four dimensional cosmological constant, $T$ is the brane tension, $k$ is a nonlocal coupling constant [31,33].

For further investigation let us rewrite our action in dimensionless space-time variables

$$
S=\int d^{4} x \sqrt{-g}\left(\frac{m_{p}^{2}}{2} R+\frac{\xi^{2}}{2} \phi \square_{g} \phi+\frac{1}{2} \phi^{2}-V(\Phi)-\Lambda^{\prime}-T\right)
$$

where $\phi$ is a dimensionless scalar field, $\Phi=e^{k \square_{g}} \phi$ and $m_{p}^{2}=g_{4} \frac{M_{p}^{2}}{M_{s}^{2}}$.

As a particular metric we take FRW one

$$
d s^{2}=-d t^{2}+a^{2}(t)\left(d x_{1}^{2}+d x_{2}^{2}+d x_{3}^{2}\right)
$$

We will consider spatially homogeneous configurations for which Beltrami-Laplace operator takes the form $\square_{g}=-\partial_{t}^{2}-3 H(t) \partial_{t}$, for the convenience of numerical calculations let us introduce the following notation $\mathcal{D}_{\mathrm{H}}^{2} \equiv \partial_{t}^{2}+3 H(t) \partial_{t}$, which we will understand as generalization of second order time derivative, which contains extra term $3 H(t) \partial_{t}$ associated with gravitational background.

Equation of motion for the space homogeneous configurations for the scalar field $\Phi$ takes the form

$$
\left(-\xi^{2} \mathcal{D}_{\mathrm{H}}^{2}+1\right) e^{2 k \mathcal{D}_{\mathrm{H}}^{2} \Phi=V^{\prime}(\Phi) .}
$$


The Friedmann equations have the following form

$$
\begin{aligned}
3 H^{2} & =\frac{1}{m_{p}^{2}} \mathcal{E}, \\
3 H^{2}+2 \dot{H} & =-\frac{1}{m_{p}^{2}} \mathcal{P} .
\end{aligned}
$$

For the case of nonlocal potentials the energy and the pressure have additional nonlocal terms $\mathcal{E}_{n l 1}$ and $\mathcal{E}_{n l 2}$

$$
\begin{aligned}
& \mathcal{E}=\underbrace{\mathcal{E}_{k}+\mathcal{E}_{p}+T+\Lambda^{\prime}}_{\text {usual local energy expression }}+\mathcal{E}_{n l 1}+\mathcal{E}_{n l 2}, \\
& \mathcal{P}=\underbrace{\mathcal{E}_{k}-\mathcal{E}_{p}-T-\Lambda^{\prime}}_{\text {usual local pressure expression }}-\mathcal{E}_{n l 1}+\mathcal{E}_{n l 2},
\end{aligned}
$$

where

$$
\begin{gathered}
\mathcal{E}_{k}=\frac{\xi^{2}}{2}(\partial \phi)^{2}, \quad \mathcal{E}_{p}=-\frac{1}{2} \phi^{2}+V(\Phi), \\
\mathcal{E}_{n l 1}=k \int_{0}^{1} d \rho\left(e^{-k \rho \mathcal{D}_{\mathrm{H}}^{2}} V^{\prime}(\Phi)\right)\left(\mathcal{D}_{\mathrm{H}}^{2} e^{k \rho \mathcal{D}_{\mathrm{H}}^{2}} \Phi\right), \\
\mathcal{E}_{n l 2}=-k \int_{0}^{1} d \rho\left(\partial e^{-k \rho \mathcal{D}_{\mathrm{H}}^{2}} V^{\prime}(\Phi)\right)\left(\partial e^{k \rho \mathcal{D}_{\mathrm{H}}^{2} \Phi}\right) .
\end{gathered}
$$

As we can see the structure of Friedmann equations and of equation for scalar field in this model is rather complicated and their study is already a very interesting mathematical problem and can be considered as a separate mathematical investigation.

To avoid calculation of $e^{-k \rho \mathcal{D}_{\mathrm{H}}^{2}}$ term which is much harder to compute than $e^{k \rho \mathcal{D}_{\mathrm{H}}^{2}}$ $(k>0)$ as computation of the former results in an ill-posed problem [35] we will use the following representation for nonlocal energy terms on the equation of motion for the scalar field

$$
\begin{aligned}
& \mathcal{E}_{n l 1}=k \int_{0}^{1} d \rho\left(\left(-\xi^{2} \mathcal{D}_{\mathrm{H}}^{2}+1\right) e^{(2-\rho) k \mathcal{D}_{\mathrm{H}}^{2} \Phi}\right)\left(\mathcal{D}_{\mathrm{H}}^{2} e^{k \rho \mathcal{D}_{\mathrm{H}}^{2} \Phi}\right), \\
& \mathcal{E}_{n l 2}=-k \int_{0}^{1} d \rho\left(\partial\left(-\xi^{2} \mathcal{D}_{\mathrm{H}}^{2}+1\right) e^{(2-\rho) k \mathcal{D}_{\mathrm{H}}^{2} \Phi}\right)\left(\partial e^{k \rho \mathcal{D}_{\mathrm{H}}^{2} \Phi}\right) .
\end{aligned}
$$

\section{Method for Solution of Nonlocal Friedmann Equations}

\subsection{Iterative Procedure for Solution Construction}

For numerical calculations let us rewrite our system in the following form

$$
3 H^{2}=\frac{1}{m_{p}^{2}} \mathcal{E},
$$




$$
\begin{gathered}
\dot{H}=-\frac{1}{2 m_{p}^{2}}(\mathcal{P}+\mathcal{E}), \\
\left(-\xi^{2} \mathcal{D}_{\mathrm{H}}^{2}+1\right) e^{2 k \mathcal{D}_{\mathrm{H}}^{2}} \Phi=V^{\prime}(\Phi) .
\end{gathered}
$$

In this paper we would like to point out which physical results we can extract from solutions of this system. First, we will find Hubble function and the scalar field from equations (15b) and (15c). It happens that equation (15a) plays a role of energy conservation from which the effective cosmological constant is extracted and is unique for every field configuration.

Integrating equation (15b) we get the following system

$$
\begin{aligned}
& \left(-\xi^{2} \mathcal{D}_{\mathrm{H}}^{2}+1\right) e^{2 k \mathcal{D}_{\mathrm{H}}^{2}} \Phi=V^{\prime}(\Phi), \\
& H=-\frac{1}{m_{p}^{2}} \int_{0}^{t} d \tau\left[\frac{\xi^{2}}{2}(\partial \phi)^{2}-k \int_{0}^{1} d \rho\left(\partial\left(-\xi^{2} \mathcal{D}_{\mathrm{H}}^{2}+1\right) e^{(2-\rho) k \mathcal{D}_{\mathrm{H}}^{2} \Phi}\right)\left(\partial e^{k \rho \mathcal{D}_{\mathrm{H}}^{2} \Phi}\right)\right] \text {. }
\end{aligned}
$$

To find solutions of the system (16) we construct the following iterative process

$$
\Phi=\lim _{n \rightarrow \infty} \Phi_{n}, \quad H=\lim _{n \rightarrow \infty} H_{n},
$$

where iterations $\Phi_{n}$ and $H_{n}$ are in turn obtained as limits of sub-iterations

$$
\Phi_{n+1}=\lim _{m \rightarrow \infty} \Phi_{n, m}, \quad H_{n+1}=\lim _{m \rightarrow \infty} H_{n, m},
$$

which are recursively defined as $(m \geqslant 0)$

$$
\begin{aligned}
\Phi_{n, m+1}= & V^{\prime-1}\left(\left(-\xi^{2} \mathcal{D}_{\mathrm{H}_{n}}^{2}+1\right) e^{2 k \mathcal{D}_{\mathrm{H}_{n}}^{2} \Phi_{n, m}}\right), \\
H_{n, m+1}= & -\frac{1}{m_{p}^{2}} \int_{0}^{t} d \tau\left[\frac{\xi^{2}}{2}\left(\partial e^{k \mathcal{D}_{\mathrm{H}_{n, m}}^{2}} \Phi_{n+1}\right)^{2}-\right. \\
& \left.k \int_{0}^{1} d \rho\left(\partial\left(-\xi^{2} \mathcal{D}_{\mathrm{H}_{n, m}}^{2}+1\right) e^{(2-\rho) k \mathcal{D}_{\mathrm{H}_{n, m}}^{2} \Phi_{n+1}}\right)\left(\partial e^{k \rho \mathcal{D}_{\mathrm{H}_{n, m}}^{2} \Phi_{n+1}}\right)\right],
\end{aligned}
$$

where initial iteration in $m$ is taken as

$$
\Phi_{n, 0}=\Phi_{n}, \quad H_{n, 0}=H_{n} .
$$

By $V^{\prime-1}$ we denote a function inverse to $V^{\prime}$. Note how sub-iteration $\Phi_{n, m}$ depends only on $H_{n}$, i.e. the whole step of sub-iterations for $H$. Note also that $H_{n, m}$ depends on $\Phi_{n+1}$, this is essentially a way to accelerate convergence.

Intuitively this iteration procedure does the following. First fixing $H_{n}$ it finds $\Phi_{n+1}$ using iteration process (17c) which is a natural generalization of a process which is described in [36-38] and for which convergence is proved analytically. Then $H_{n+1}$ is obtained by iterating (17d) where $\Phi_{n+1}$ is fixed as computed in the previous step. Thus found $H_{n+1}$ is used to find $\Phi_{n+2}$ and so on. This kind of multi-step iterative procedure are known to provide rapid convergence. In our experiments this iterative procedure converges much more reliably as compared to more naive iterative procedure where one does not do intermediate iterations.

In a similar fashion one can construct iterative procedures for systems with several scalar fields such as those studied in $[35,39]$. 


\subsection{Exponentiating $\mathcal{D}_{\mathrm{H}}^{2}$ by Solving Diffusion Equation}

Equations of motion as well as the iteration method discussed above require computation of action with operator $e^{k \rho \mathcal{D}_{\mathrm{H}}^{2}}$ on a given function. Computationally this is a nontrivial problem by itself. We use the following method which beside giving mathematical definition for such an operator more importantly provides corresponding computational method.

Result of the acting with operator $e^{\rho \mathcal{D}_{\mathrm{H}}^{2}}$ on a given function $\varphi(t)$ is natural to define as a solution of the following diffusion partial differential equation with corresponding initial and boundary conditions

$$
\begin{aligned}
\frac{\partial}{\partial \rho} f(\rho, t) & =\mathcal{D}_{\mathrm{H}}^{2} f(\rho, t), \\
f(0, t) & =\varphi(t), \\
f(\rho, \pm \infty) & =\varphi( \pm \infty),
\end{aligned}
$$

where $-\infty<t<+\infty, \rho \geqslant 0$. Note that here evolution goes along $\rho$ axis, while $t$ plays a role of space. Solution of $(18)$ is a rigorous mathematical definition of the operator $e^{\rho \mathcal{D}_{\mathrm{H}}^{2}}$ acting on function $\varphi$

$$
e^{\rho \mathcal{D}_{\mathrm{H}}^{2}} \varphi(t)=f(\rho, t) .
$$

Such solution exists and is unique for wide classes of $H$ and $\varphi$ [40]. Since in standard settings such a diffusion problem is well defined in particular for $H, \varphi \in \mathfrak{L}_{2}(\mathbb{R})$ which seem to suffice applications which are considered here we will not discuss this topic any further.

\subsection{Fixing Potential and Other Parameters}

So far we did not specify exact shape of the potential for our system. In this paper we are interested in coupled to the gravity a tachyon field which appears as a lowest excitation in level truncation scheme for fermionic string field theory [31]. Thus all further discussion will be about quartic potential

$$
V(\Phi)=\frac{1}{4} \Phi^{4}
$$

We would like to note though that from numerical perspective this potential can lead to difficulties as it results in solutions which are not differentiable at zero (they behave as $t^{1 / 3}$ at in the vicinity of zero [41]). For the later reason we found it useful to study similar problem in potential

$$
V_{\alpha}(\Phi)=(1-\alpha) \frac{1}{2} \Phi^{2}+\alpha \frac{1}{4} \Phi^{4}, \quad 0<\alpha<1
$$

As $\alpha \rightarrow 1$ this potential tends to potential (21). The interesting property of this potential is that solution has finite derivative at zero and moreover there is a theorem which states convergence of our type of iterative procedure in case of Minkowski metric [42]. 
Let us return to the potential (21). Equation (15c) is invariant under shifts

$$
\Phi(t) \rightarrow \Phi\left(t+t_{0}\right), \quad H(t) \rightarrow H\left(t+t_{0}\right),
$$

and mirroring

$$
\Phi(t) \rightarrow-\Phi(-t), \quad H(t) \rightarrow-H(-t) .
$$

Thus it is natural to look for odd solutions. Without loss of generality we can set $\Phi(0)=0$.

Equation (15c) has the following constant solutions

$$
\Phi=0, \pm 1 .
$$

Following the same logic as in Minkowsky case $[31,36]$ since $V(\Phi=+1)=V(\Phi=-1)$ we can expect existence of kink-type solutions where scalar field interpolates between $\Phi=+1$ and $\Phi=-1$.

Iterative procedure (17) requires initial iteration $\Phi_{0}, H_{0}$ to be specified explicitly. To find such kink solutions it is natural to take

$$
\Phi_{0}(t)=\operatorname{sign}(t), \quad H_{0}(t)=0 .
$$

This initial iteration leads to a rapid convergence. It is interesting that our numerical experiments show that iterative procedure (17) converges to a kink-type solution if we start from any two bounded functions which are positive on positive semi-axis and negative on negative one. This observation provides a strong indication for uniqueness of the solutions (up to shifts and mirroring).

The value of $\xi^{2}$ is determined during level-truncation procedure, its value is

$$
\xi_{\text {phys }}^{2}=-\frac{1}{4 \log \frac{4}{3 \sqrt{3}}} \approx 0.96 .
$$

Nevertheless since different values of $\xi^{2}$ already in Minkowski case result in different physical pictures we will systematically consider various values of $\xi^{2}$. Note also that the case $\xi^{2}=0$ corresponds to $p$-adic string with $p=3$. The value of nonlocal coupling constant is fixed by the same procedure it is $k=\frac{1}{8}$ in our case.

\section{Numerical Results}

\subsection{Numerical Solutions for Friedmann Equations}

In what follows for numerical computations we put $m_{p}=1$. We used iterative method presented in the previous section to solve Friedmann equations for our system. We observe the crucial role of the value of $\xi^{2}$, as it varies the following physical properties of the system are affected (see Fig!1). 
- For $\xi^{2}=0$ the solution in both $\Phi$ and $H$ has a monotonic kink shape. This behavior is qualitatively similar to the case of Minkowsky metric [31,37,43].

- There exists a critical value $\xi_{\text {osc }}^{2} \approx 1.18$ which determines between types of the $\Phi$ component of the solution - kink or oscillatory with finite period. For $\xi^{2}<\xi_{\text {osc }}^{2}$ the solution has a kink shape with exponentially decreasing oscillations around \pm 1 as $t \rightarrow \pm \infty$. On the other hand if $\xi^{2}>\xi_{\text {osc }}^{2}$ the solution converts to oscillations with finite period 1 Similar behavior was observed in the case of Minkowsky metric, although in that case the critical value was higher $\approx 1.38[31,37]$.

- There is one more critical value $\xi_{\text {shape }}^{2} \approx 0.42$ which determines a shape of a Hubble function (see Fig 2). More precisely, as $\xi^{2}$ grows Hubble function $H$ gets a turning point at some positive time $t_{0}$. Moreover, for $\xi^{2}>\xi_{\text {shape }}^{2} H$ ends up in tending a negative (positive) value as $t \rightarrow \infty(t \rightarrow-\infty)$. This is a new type of behavior which was not present in Minkowsky case.

Note that the physical value of $\xi^{2}(24)$ is in the region

$$
\xi_{\text {shape }}^{2}<\xi_{\text {phys }}^{2}<\xi_{\text {osc }}^{2} \text {. }
$$

Figures 1, 2 also show how dynamics of the scale factor $a(t)$

$$
a(t)=a_{0} \exp \left(\int_{0}^{t} H(\tau) d \tau\right)
$$

changes as $\xi^{2}$ increases. For $\xi^{2}=0$ it has a has a minimum at the perturbative vacuum $\phi=0$ and increases as $\phi$ tends to nonperturbative vacua \pm 1 . As $\xi^{2}$ increases the shape of $a$ changes and for $\xi^{2}>\xi_{\text {shape }}^{2}$ the scale factor decreases as $\phi \rightarrow \pm 1(t \rightarrow \pm \infty)$. For numerical computations we use initial condition $a_{0}=1$ in (25).

\subsection{Two Profiles of the Hubble Function}

To analyze the late time behavior of Hubble function let us use the so called mechanical approximation which was studied for example in [14] and gives realistic qualitative picture at least for the late time behavior. Mechanical approximation is constructed by keeping in original nonlocal expression only terms with derivatives no higher than second order. For $(16 \mathrm{~b})$ we get

$$
H(t) \approx-\frac{1}{m_{p}^{2}} \int_{0}^{t} d \tau\left(\frac{\xi^{2}}{2}(\partial \phi)^{2}-k(\partial \phi)^{2}\right)=\frac{1}{m_{p}^{2}} \int_{0}^{t} d \tau\left(k-\frac{\xi^{2}}{2}\right)(\partial \phi)^{2}
$$

\footnotetext{
1 Note that for iterative method which we present in this paper it is essential that solution $\Phi$ has well defined limits at infinite times. This fact is used in particular for the boundary condition (20). This means that this method is not suitable for finding oscillatory solutions with high precision. Oscillatory behavior reported in this paper was obtained by vastly enlarging the lattice in $t$, this did not allow finding critical value of $\xi^{2}$ with more then 3 significant digits. Reliable methods for finding oscillatory solutions for the type of equations considered in this paper is an open mathematical problem even for Minkowski metric, see [37]. Note though that physically significant solutions in this model turned out to be of kink type where iterations rapidly converge.
} 


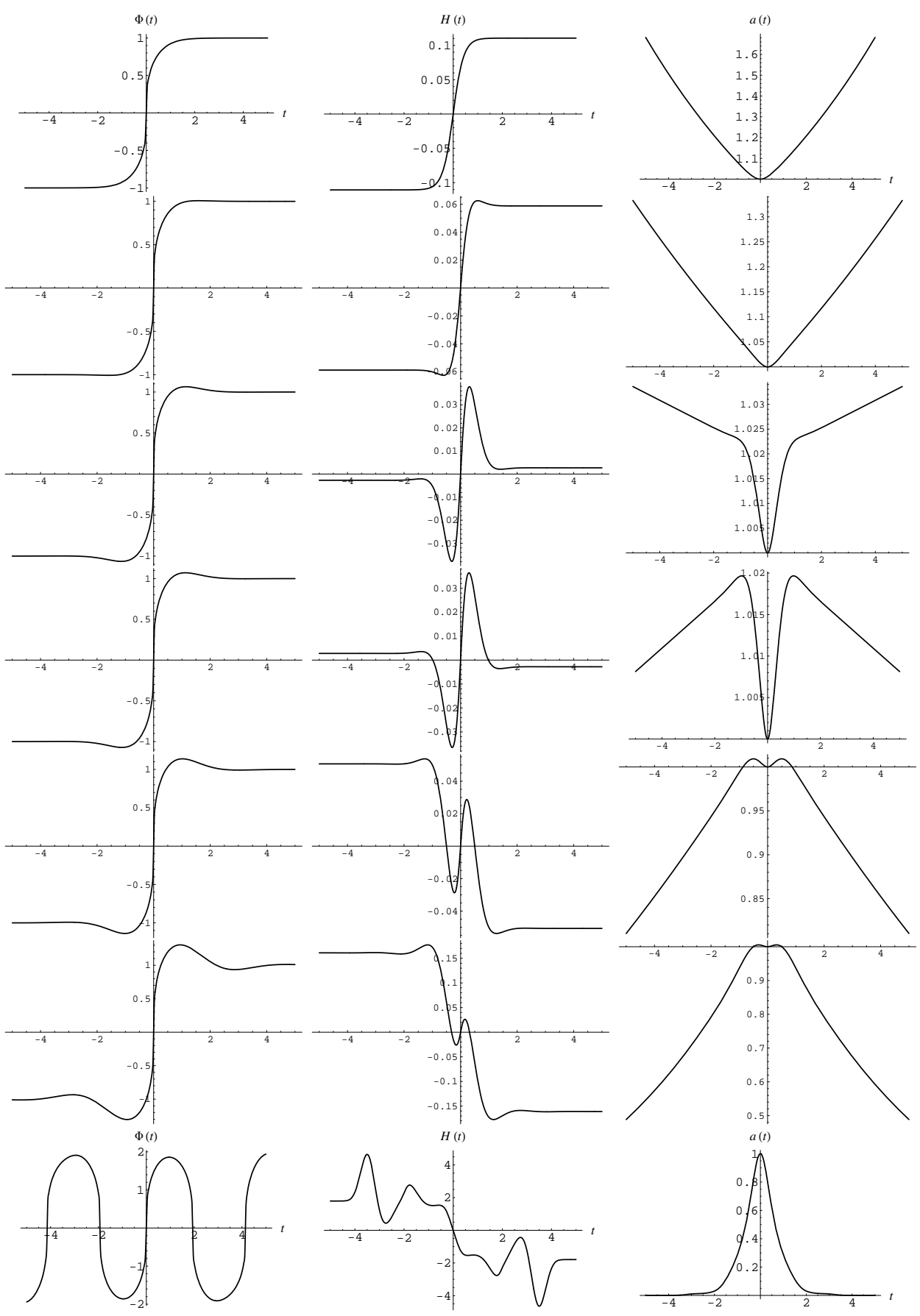

Figure 1: Solutions of the Friedmann equations $\Phi, H$ and $a$ (left to right) for different values of the parameter $\xi^{2}=0,0.2,0.41,0.43,0.6,0.96,2$ (top to bottom). Note that on the figures for scale factor $a(t)$ axes cross at the point $(t=0, a=1)$. We can see that when $\xi^{2}$ increases the shape of the scale factor changes from parabolic type $(a(t) \geqslant 1$ for all times) to lump type $(a(t) \leqslant 1$ for all times); transition between these shape types is illustrated on the Fig. 2. 


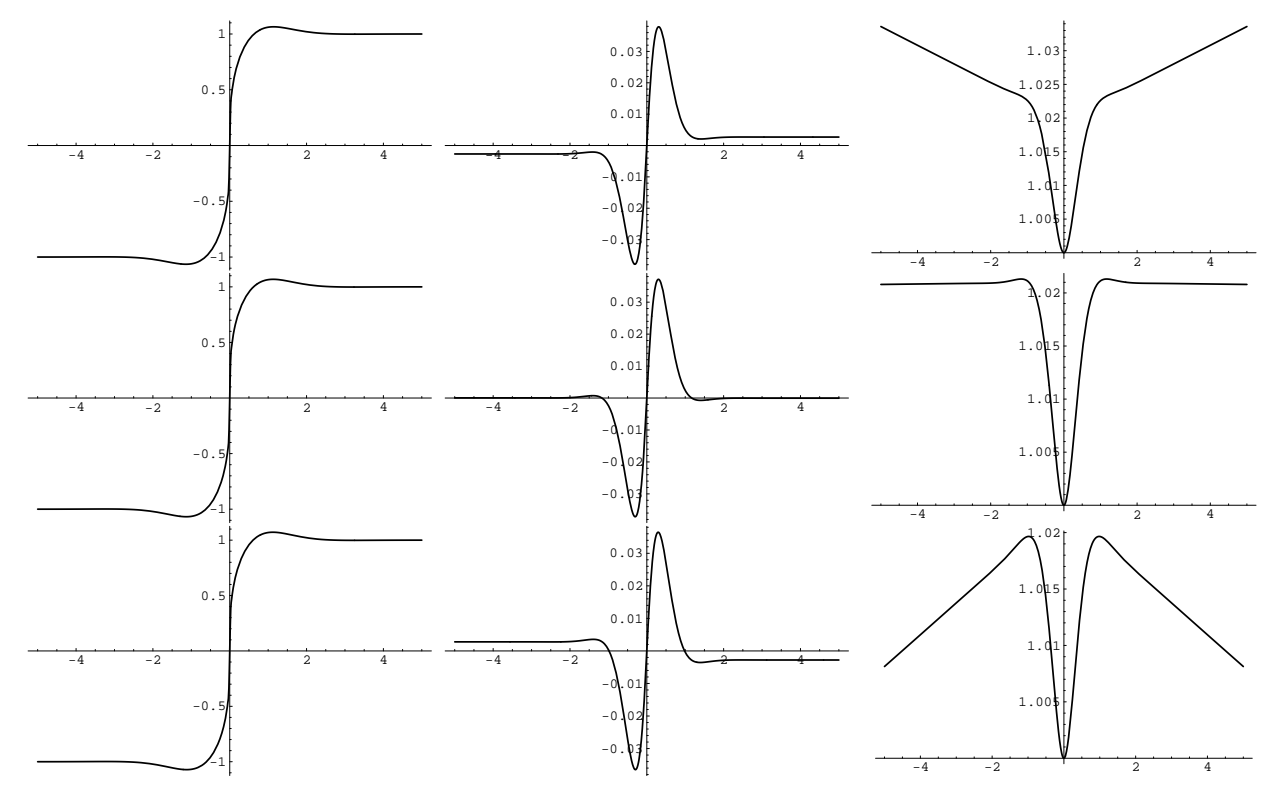

Figure 2: Functions $\Phi, H$ and $a$ (left to right) for $\xi^{2}=0.41,0.42,0.43$ (top to bottom). Note how asymptotic behavior changes as we go through $\xi^{2}=0.42$.

We can see from the expression above that there exists a value of $\xi^{2}$ which determines the change of sign for the Hubble function, more precisely for $\xi^{2}<2 k$ the Hubble function is positive for $t \rightarrow \infty$ (negative as $t \rightarrow-\infty$ ), while for $\xi^{2}>2 k$ the reverse becomes true. Thus mechanical approximation gives us the critical value of $\xi^{2}=2 k=0.25$. As already described numerical computations give us the value $\xi_{\text {shape }}^{2} \approx 0.42$ which slightly differs from the just found approximate critical $\xi^{2}$. This difference manifests the influence of higher order nonlocal terms. Nevertheless the analysis above clarifies the phenomenon which causes the change of sign for the asymptotic values of the Hubble function.

\subsection{Two Regimes of the Solution}

In this section we will address the mechanism which forms numerically found exponentially decreasing oscillations and the existence of critical value $\xi_{\text {osc }}^{2}$. We will also compare this behavior with results obtained without gravitational term in action [37].

Let us present solution of equation (15c) for the quartic potential $V(\Phi)=\frac{1}{4} \Phi^{4}$

$$
\left(-\xi^{2} \mathcal{D}_{\mathrm{H}}^{2}+1\right) e^{2 k \mathcal{D}_{\mathrm{H}}^{2}} \Phi=\Phi^{3}
$$

as a sum

$$
\Phi(t)=\Phi_{0}(t)+\chi(t),
$$

where $\Phi_{0}$ denotes solution of equation (15c) for $\xi^{2}=0$. Substituting (28) to (15c) and leaving only linear terms in $\chi$ we get

$$
\left(-\xi^{2} \mathcal{D}_{\mathrm{H}}^{2}+1\right) e^{2 k \mathcal{D}_{\mathrm{H}}^{2}}\left(\Phi_{0}+\chi\right)=\Phi_{0}^{3}+3 \Phi_{0}^{2} \chi
$$


Using the fact that $\Phi_{0}$ is the solution of equation (15c) for $\xi^{2}=0$ we can write equation for $\chi(t)$ which in linear approximation has the form

$$
\left(-\xi^{2} \mathcal{D}_{\mathrm{H}}^{2}+1\right) e^{2 k \mathcal{D}_{\mathrm{H}}^{2}} \chi=3 \Phi_{0}^{2} \chi+\xi^{2} \mathcal{D}_{\mathrm{H}}^{2} e^{2 k \mathcal{D}_{\mathrm{H}}^{2}} \Phi_{0} .
$$

Using the fact that operator $e^{2 k \mathcal{D}_{\mathrm{H}}^{2}}$ acts as an identity on constants we can write large $t$ approximation

$$
\left(-\xi^{2} \mathcal{D}_{\mathrm{H}}^{2}+1\right) e^{2 k \mathcal{D}_{\mathrm{H}}^{2}} \chi=3 \chi,
$$

were we used asymptotical properties of solution $\Phi_{0}$.

To carry out harmonic analysis for equation (31) we will consider eigenfunctions of the D'Alembertian operator $\mathcal{D}_{\mathrm{H}}^{2} v_{\lambda}=-\lambda v_{\lambda}$. Using that $e^{2 k \mathcal{D}_{\mathrm{H}}^{2}} v_{\lambda}=e^{-2 k \lambda} v_{\lambda}$ and expanding $\chi$ in $v_{\lambda}$ equation (31) leads us to

$$
\left(\xi^{2} \lambda+1\right) e^{-2 k \lambda} v_{\lambda}=3 v_{\lambda}
$$

Our goal is to analyze which values of $\xi^{2}$ allow real-valued $\lambda$, i.e. non-damping oscillations take place. Considering equation (32) as an equation for complex variable $\lambda$ with parameter $\xi^{2}$ we obtain that there is a minimum value of $\xi_{0}^{2} \approx 1.77$ for which $\lambda$ is real. So for $\xi^{2}<\xi_{0}^{2}$ we have solutions with nonzero imaginary parts which result in vanishing solutions while $\xi^{2}>\xi_{0}^{2}$ leads to oscillatory regime. Equation (32) has exactly the same form in case of Minkowsky space which was considered earlier [37] (functions $v_{\lambda}$ are different as they depend on $H$ ). As we can see method discussed in this section provides us only qualitative explanation of the changing of the regimes, the value of critical $\xi^{2}$ found here only approximately reproduces numerically obtained $\xi_{\text {osc }}^{2} \approx 1.18$. It is interesting to note that Minkowski case leads to higher value of critical $\xi^{2}$ [37], i.e. it appears that "friction" $H(t)$ does not damp oscillations but au contraire increases them.

\section{Cosmological properties}

It is interesting to note, that initially we considered a model with potential $V(\phi)=$ $-\frac{1}{2} \phi^{2}+\frac{1}{4} \phi^{4}+\frac{1}{4}$, see Fig. 3a). Nonlocal term contributes to kinetic term, most importantly
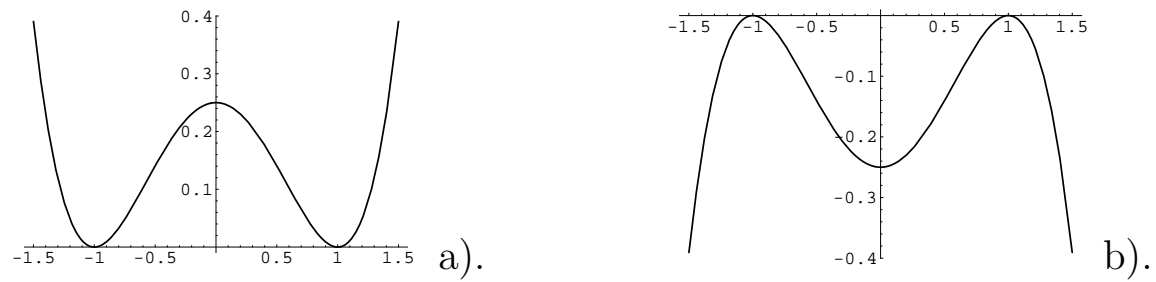

Figure 3: $W$ and $-W$ shaped potentials.

it changes its sign [11], or equivalently changes the sign of potential (kinetic term is positive in this case), see Fig.3. In this context it is interesting to study already mentioned mechanical approximation which can illustrate such an effect. 
Solutions of (15) lead to interesting cosmological properties. Let us consider them for physically interesting values of $\xi^{2}$ and see what kind of physical behavior they result in. We would like to study dynamics of the following physical quantities. The state parameter which is defined as usual

$$
w=\frac{p}{\rho}
$$

or in the terms of Hubble parameter

$$
w=-1-\frac{2}{3} \frac{\dot{H}}{H^{2}}
$$

will be presented on the figures below along with deceleration parameter defined as

$$
q(t)=-\frac{\ddot{a} a}{\dot{a}}
$$

\subsection{Effective mechanical potential}

In order to better understand which behavior of the system we might expect let us first study equation (9) in local approximations.

If we ignore the nonlocal operator $e^{2 k \mathcal{D}_{\mathrm{H}}^{2}}$ in (9) we get

$$
\left(-\xi^{2} \mathcal{D}_{\mathrm{H}}^{2}+1\right) \Phi=\Phi^{3}
$$

Let us reproduce action which leads to the equation above

$$
S=\int d^{4} x \sqrt{-g}\left(\frac{m_{p}^{2}}{2} R+\frac{\xi^{2}}{2} \Phi \square_{g} \Phi+\frac{1}{2} \Phi^{2}-\frac{1}{4} \Phi^{4}\right) .
$$

This action describes a particle moving in the system with friction $H(t)$

$$
\begin{gathered}
\xi^{2} \partial^{2} \Phi=\Phi-\Phi^{3}-3 H \xi^{2} \partial \Phi \\
\dot{H}=-\frac{\xi^{2}}{m_{p}^{2}}(\partial \Phi)^{2}
\end{gathered}
$$

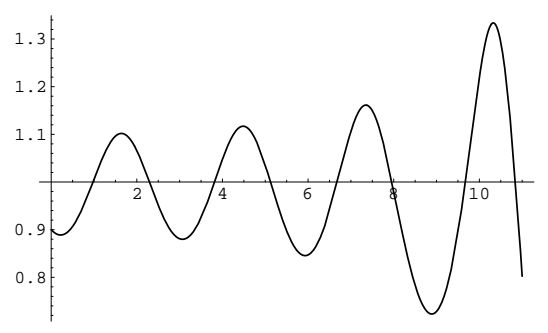

a).

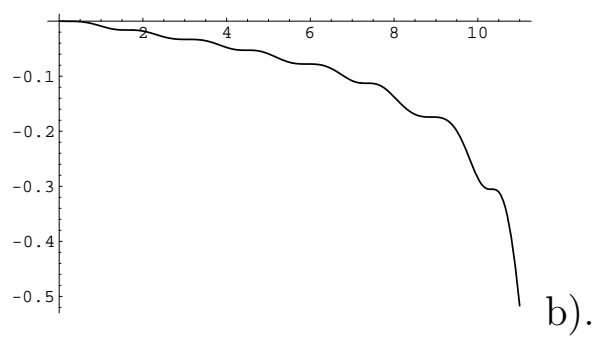

Figure 4: Anharmonic oscillator with W-shape potential; a) periodic scalar field trajectory located around $\Phi=1$; b) the solution for the Hubble function $\mathrm{H}(\mathrm{t}), \xi^{2}=0.4$. 

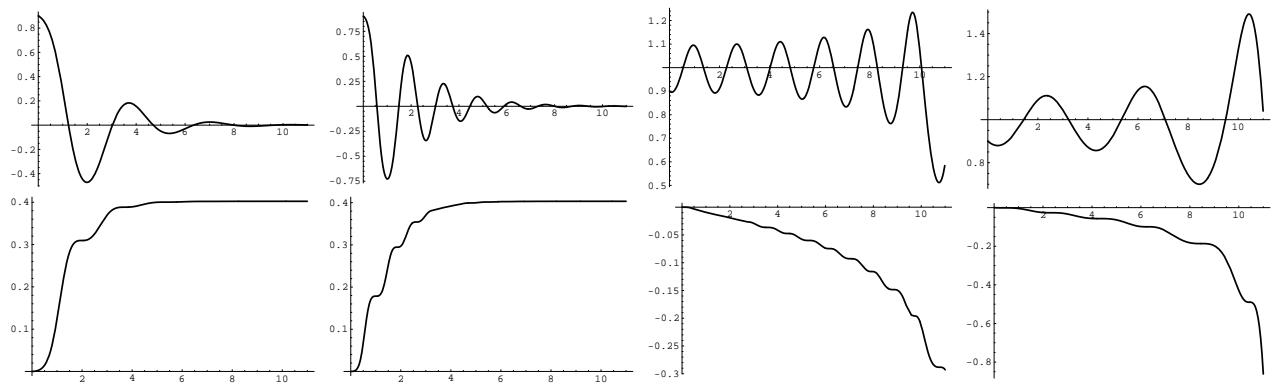

Figure 5: Scalar field $\Phi(t)$ (upper row) and Hubble function $H(t)$ (lower row) for the system (41) for different values for $\xi^{2}$ parameter $\xi^{2}=0,0.2,0.4,1$ from left to right correspondingly.

As we can see here Hubble function is explicitly negative and thus we obtained a system which is unusual from mechanical point of view - it is an anharmonic oscillator with negative friction (see Fig. (4). Such a behavior looks similar with the behavior for nonlocal systems studied in $[14,15]$ in the Minkowski background, when the trajectories of the scalar field exceed the extremum of the potential, meanwhile the energy is conserved.

Now let us consider mechanical approximation by accounting in the equation (9) only derivatives no higher that second order, we get

$$
\left(\left(2 k-\xi^{2}\right) \partial^{2}+1\right) \Phi=\Phi^{3}-3\left(2 k-\xi^{2}\right) H(t) \partial \Phi .
$$

Equation (39) could be obtain from the following action

$$
S=\int d^{4} x \sqrt{-g}\left(\frac{m_{p}^{2}}{2} R+\frac{\left(\xi^{2}-2 k\right)}{2} \Phi \square_{g} \Phi+\frac{1}{2} \Phi^{2}-\frac{1}{4} \Phi^{4}\right),
$$

which leads to the following local Friedmann equations

$$
\begin{gathered}
\partial^{2} \Phi=-\frac{\Phi-\Phi^{3}}{\left(2 k-\xi^{2}\right)}-3 H \partial \Phi, \\
\dot{H}=\frac{\left(2 k-\xi^{2}\right)}{m_{p}^{2}}(\partial \Phi)^{2} .
\end{gathered}
$$

This system of equations describes particle moving in the following potential

$$
V(\Phi)=\frac{2 \Phi^{2}-\Phi^{4}}{4\left(2 k-\xi^{2}\right)} .
$$

As we can see for $\xi^{2}<2 k$ flip of the potential takes place. In particular, for $\xi^{2}=0$ we get an equation describing particle moving in the $-W$-shape potential (see Fig.3b). For $\xi^{2}>2 k$ the potential has $W$-shape (see Fig 3 a). This simple remark leads to important physical consequences, we might expect two different regimes of the solution. Solutions for scalar field and Hubble function for different values of $\xi^{2}$ are presented on Fig 5 . We 
see two regimes - decreasing oscillations with positive Hubble function and increasing oscillations with positive Hubble function.

It is important to note that while mechanical approximation helps us to illustrate or explain qualitatively some physical consequences, it is a rather rough approximation and does not necessarily capture some nonlocal effects. This situation is already depicted by the fact that in full nonlocal model and its mechanical approximation the change of regime of the solution corresponding to $W$ and $-W$ shapes of the potential happens for different values of $\xi^{2}$, they are of the same order though.

\subsection{Rolling tachyon in the FRW background and the effective cosmological constant $\Lambda^{\prime}$}

Let us study the value of effective cosmological constant $\Lambda^{\prime}$. Formally $\Lambda^{\prime}$ enters action as a correction of $D 3$-brane tension $T$. For rolling solution in Minkowski background it is known $[11,31,44]$ that the value of $T$ is essential for calculating stress tensor, but as it does not enter equations of motion it is not essential for the existence of solution. Moreover according to Sen's conjecture [11,31,44]

$$
T_{\text {Minkowski }}=-V(\Phi= \pm 1)=\frac{1}{4} \text {. }
$$

Such value of $T$ in Minkowski background corresponds to zero cosmological constant in the non-perturbative vacuum $\Phi= \pm 1$.

In case of FRW metric we represent $D 3$-brane tension as $T=T_{\text {minkowski }}+\Lambda^{\prime}$ where $\Lambda^{\prime}$ is determined uniquely for each field configuration (situation is the same in local theories). Note that $\Lambda^{\prime}$ does not enter equations (16), in fact we determine its value from (15a). Different values of $\Lambda^{\prime}$ for different $\xi$ are presented on table 1. Note that we worked with dimensionless variables. If we return to initial notations and take into account physical constants in action (6) we can get realistic value of $\Lambda$ because generally speaking the string scale does not coincide with the Plank mass $[11,17,19]$.

\begin{tabular}{|c|c|}
\hline$\xi^{2}$ & $\Lambda^{\prime}$ \\
\hline 0 & 0.036938 \\
\hline 0.2 & 0.010379 \\
\hline 0.41 & 0.000022 \\
\hline 0.42 & 0. \\
\hline 0.43 & 0.000023 \\
\hline 0.6 & 0.007766 \\
\hline 0.96 & 0.077848 \\
\hline
\end{tabular}

Table 1: Values of $\Lambda^{\prime}$ for different $\xi^{2}$. 

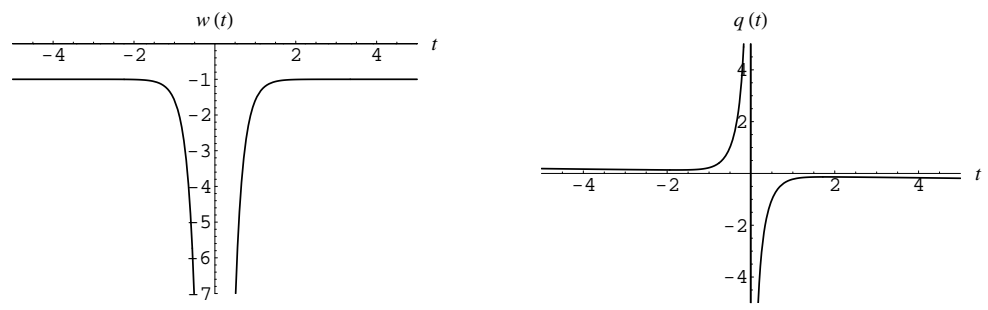

Figure 6: The state and deceleration parameters for the case of $p$-adic cosmological model $\left(\xi^{2}=0\right)$.

\section{3 $\quad P$-adic cosmological model, $\xi^{2}=0$}

Recently $p$-adic string models started to attract a lot of attention being used as toy cosmological models. In [19] $p$-adic string model [31] was considered in the context of inflation and approximate solutions of the fully nonlocal $p$-adic string theory coupled to gravity were constructed. In section 3.1 we presented a new algorithm for construction of precise solutions for these models. While iterative procedure of section 3.1 converges for any odd $p$ (for rigorous mathematical proof for the simpler Minkowski case see [43]) here we will analyze cosmological properties of the solution for the case $p=3$. This case is specifically interested since as mentioned earlier it corresponds to zero mass of the tachyon field, $\xi^{2}=0$, in level truncated fermionic string field theory (SFT).

We can see on Fig,1 and Fig, 6 that scalar field and background solutions are monotonic functions which tend to constants as $t \rightarrow \pm \infty$. Since these solutions do not have turning points (oscillations) there is no crossing of the cosmological state parameter barrier $(w=$ -1 ), it is negative and approaches to -1 from below (Fig [6). For these solutions we have nonsingular accelerating Universe with a bounce. As an illustration we can note that behavior of the scale factor obtained could be well approximated by $a_{\text {approx }}(t)=e^{H_{0} t}$ for large $t>0$ and by $a_{\text {approx }}(t)=e^{-H_{0} t}$ for large $t<0$, where $H_{0}=\lim _{t \rightarrow \infty} H(t)$. Thus we get de Sitter type solution for flat FRW background for $t \rightarrow+\infty$ and anti de Sitter as $t \rightarrow-\infty$. It might be useful to remark that bouncing cosmology is a subject of of recent investigation which include the Ekpyrotic [34], Pre-Big-Bang [45] and higher derivative modification of Einstein gravity scenarios [46].

Deceleration parameter $q$ is negative and approaches -1 from below as $t \rightarrow \pm \infty$.

\subsection{SFT cosmological model, $\xi^{2}=\xi_{\text {phys }}^{2}$}

Today SFT is the strongest candidate for real physical theory of nature. As we mentioned earlier level truncation in non-sypersymmetric fermionic SFT leads to action (17) with potential (21) with $\xi^{2}=\xi_{\text {phys }}^{2}$ (24) $[11,31,33]$. The algorithm presented in section 3.1 allowed us to study this model coupled to FRW backgroud without explicit approximations.

As shown on Fig:1 and Fig.7 in this case scalar field solution has turning points (decreasing oscillations) and tends to stationary solutions \pm 1 as $t \rightarrow \pm \infty$. The shape of Hubble function is essentially different as compared to the case $\xi^{2}=0$, indeed it has a 

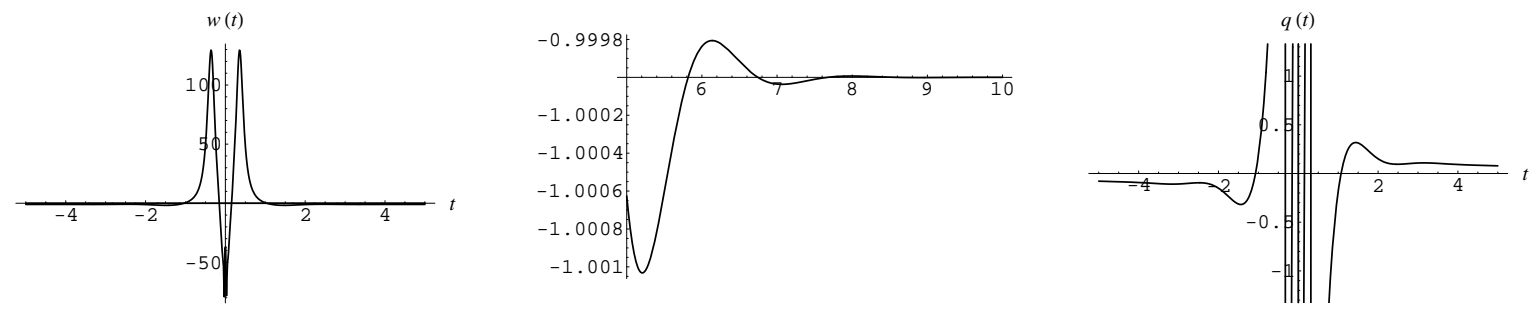

Figure 7: The state parameter, its fine structure and deceleration parameter for the case of SFT cosmological model $\left(\xi^{2}=\xi_{\text {phys }}^{2}\right)$.

clear maximum and tends to negative constant for $t>0, \lim _{t \rightarrow \pm \infty} H(t)=\mp 0.15$. Such behavior is in agreement with analysis presented in the previous section since $\xi_{\text {phys }}^{2}>\xi_{\text {osc }}^{2}$.

The state parameter $w(t)$ tends to -1 as $t \rightarrow \pm \infty$ and has exponentially decreasing oscillations around $w=-1$ barrier. Note that models which allow for crossing of $w=-1$ barrier are a subject of many recent investigations [17, 47-53]. Here we observe this behavior in nonlocal model with only one scalar field as in [17]. We also see that we have a decelerating phase at late times, this property was not predicted by approximate methods [11].

\section{Conclusions}

In this paper we have studied the dynamics of nonlocal cosmological models driven by String Field Theory. These models have an infinite number of derivatives and are characterized by positive constants $k, \xi$ and $\Lambda^{\prime}$ which determine the shape of the solution. We have developed a new method for solving nonlocal Friedmann equations. Such equations contain infinite number of derivatives and form a new class of equations in mathematical physics which recently started to be discussed in literature $[15,18,19,37,41-43,54]$.

To study cosmological properties we have investigated the behavior of nonlocal models in the FRW background. We are especially interested in two physical cases: p-adic cosmological models and level-truncated SFT model. In $p$-adic cosmological model we obtained nonsingular bouncing solution with $w$ parameter approaching the -1 barrier from below. SFT model has nonsingular solution for which $w$ parameter crosses -1 barrier. We also discussed the possibility of obtaining realistic cosmological constant from considered above nonlocal cosmological models.

\section{Acknowledgements}

The author would like to thank D. Mulryne and N. Turok for very useful discussions. The author is also grateful to Ya. Volovich for the assistance with numerical calculations. The author also would like to thank I.Ya. Aref'eva and S.V. Vernov for their comments on an earlier version of this manuscript. The author gratefully acknowledge the use of the UK National Supercomputer, COSMOS, funded by PPARC, HEFCE and Silicon Graphics. 
This work is supported by the Center for Theoretical Cosmology, in Cambridge and in part by RFBR grant 05-01-00758, INTAS grant 03-51-6346 and Russian President's grant NSh-672.2006.1.

\section{References}

[1] S. Perlmutter et al.,(Supernova Cosmology Project collaboration), Astrophys. J. 517, 565 (1999); A.G. Riess et al., (Supernova Search Team collaboration), Astron. J. 116, 1009 (1998); Astrophys. J. 607, 665 (2004); R.A. Knop et al., (Supernova Cosmology Project collaboration), Astrophys. J. 598, 102 (2003); M. Tegmark et al., (SDSS collaboration), APJ 606, 702 (2004); D.N. Spergel et al., (WMAP collaboration), Astrophys. J. Suppl. Ser. 148, 175 (2003);Astrophys. J. Suppl. Ser. 170, 377 (2007); P. Astier et al., Astron. Astrophys. 447, 31 (2006); U. Seljak, A. Slosar and P. McDonald, J. Cosmol. Astropart. Phys. 10 (2006) 014.

[2] E. J. Copeland, M. Sami and Sh. Tsujikawa, Int. J. Mod. Phys. D15, 1753 (2006).

[3] T. Padmanabhan, AIP Conf. Proc. 861, 179 (2006).

[4] R.R. Caldwell, R. Dave and P.J.Steinhardt, Phys.Rev Lett 80, 1582 (1998).

[5] C. Armendariz-Picon, V. Mukhanov and P.J. Steinhardt, Phys.Rev.Lett. 85, 4438 (2000).

[6] B. Feng, X. Wang, X. Zhang, Phys.Lett. B 607, 35 (2005); H. Wei, Sh. N. Zhang, Phys. Rev. D 76, 063005 (2007); Y.-F. Cai, T. Qiu, Y.-S. Piao, gr-qc/0704.1090.

[7] G.W. Gibbons, Classical Quantum Gravity 20, S321 (2003).

[8] G. Calcagni, A. R. Liddle, Phys.Rev.D 74, 043528, (2006).

[9] M. Gasperini, arXiv:hep-th/0310293; F. Piazza, Sh. Tsujikawa, J. Cosmol. Astropart. Phys. 07 (2007) 004; E. Elizalde, S. Jhingan, S. Nojiri, S.D. Odintsov, M. Sami, I. Thongkool, arXiv:0705.1211.

[10] S. M. Carroll, V. Duvvuri, M. Trodden, M. S. Turner, Phys.Rev. D 70, 043528 (2004); A.D. Dolgov, M. Kawasaki, Phys.Lett. B 573, 1 (2003); M. E. Soussa, R. P. Woodard, Gen.Rel.Grav. 36, 855 (2004); G. Allemandi, A. Borowiec, M. Francaviglia, Phys.Rev. D70, 043524 (2004); 70, 103503 (2004); J. W. Moffat, astro-ph/0403266; J. D. Barrow, Class.Quant.Grav. 21, L79 (2004); M.C.B. Abdalla, Sh. Nojiri, S. D.Odintsov, Class.Quant.Grav. 22, L35 (2005); Sh. Nojiri, S. D. Odintsov, P. V. Tretyakov, Phys. Lett. B 651, 224 (2007); S. Deser, R. P. Woodard, Phys. Rev. Lett. 99, 111301 (2007).

[11] Irina Ya. Aref'eva, AIP Conf.Proc. 826, 301 (2006). 
[12] E. J.Copeland, M. R.Garousi, M.Sami, Sh. Tsujikawa, Phys. Rev. D 71, 043003 (2005); I. Ya. Aref'eva, A.S. Koshelev, S.Yu. Vernov, Teor. Math. Phys. (Engl. Transl.) 148, 895 (2006); M. Libanov, E. Papantonopoulos, V. A. Rubakov, M. Sami, Sh. Tsujikawa, J. Cosmol. Astropart. Phys. 08 (2007) 010.

[13] S.M. Carroll, M. Hoffman and M. Trodden, Phys. Rev. D 68, 023509 (2003); J.M. Cline, S. Jeon and G.D. Moore, Phys. Rev. D 70, 043543 (2004); S.D.H. Hsu, A. Jenkins and M.B. Wise, Phys. Lett. B 597, 270 (2004); B. McInnes, Nucl. Phys. B 718, 55 (2005); R.V. Buniy, S.D.H. Hsu, and B.M. Murray, Phys. Rev. D 74, 063518 (2006); E. O. Kahya, V. K. Onemli, Phys.Rev. D 76, 043512 (2007).

[14] I.Ya Aref'eva, L.V. Joukovskaya, J. High Energy Phys. 10 (2005) 087.

[15] V. Forini, G. Grignani, G. Nardelli, J. High Energy Phys. 03 (2005) 079.

[16] G. Calcagni, J. High Energy Phys. 05 (2006) 012.

[17] I.Ya Aref'eva, L.V. Joukovskaya, S.V. Vernov, J. High Energy Phys. 07 (2007) 087.

[18] G. Calcagni, M. Montobbio, G. Nardelli, hep-th/0705.3043 [Phys. Rev. D (to be published)].

[19] N. Barnaby, T. Biswas, J. M. Cline, J. High Energy Phys. 04 (2007) 056.

[20] N. Barnaby, J.M. Cline, arXiv:hep-th/0704.3426.

[21] J. E. Lidsey, Phys. Rev. D 76, 043511 (2007).

[22] F. C. Mena, D. J. Mulryne, R. Tavakol, Classical Quantum Gravity 24, 2721 (2007).

[23] A. J. Tolley, D. H. Wesley, J. Cosmol. Astropart. Phys. 05 (2007) 006.

[24] J.-L. Lehners, P. McFadden, N. Turok, P. J. Steinhardt, arXiv:hep-th/0702153.

[25] R.R. Metsaev, Ch. B. Thorn, A.A. Tseytlin, Nucl.Phys.B 596, 151 (2001).

[26] R.R. Metsaev, Nucl.Phys.B 625, 70 (2002).

[27] E. Witten, Nucl. Phys. B268, 253 (1986); E. Witten, Nucl.Phys. B276, 291 (1986).

[28] V. Kostelecky and S. Samuel, Phys. Rev. D 39, 683 (1989).

[29] P. West, Phys.Lett. B 548, 92 (2002).

[30] I.A. Aref'eva, P.B. Medvedev and A.P. Zubarev, Phys.Lett. B 240, 356 (1990).

[31] I.Ya. Aref'eva, L.V. Joukovskaya and A.S. Koshelev, J. High Energy Phys. 09 (2003) 012. 
[32] N.Berkovits, A.Sen, B.Zwiebach, Nucl.Phys. B587, 147 (2000).

[33] I.Ya. Arefeva, D.M. Belov, A.S. Koshelev, P.B. Medvedev, Nucl.Phys. B638, 3 (2002).

[34] J. Khoury, B.A. Ovrut, P.J. Steinhardt and N. Turok, Phys. Rev. D 64, 123522 (2001); J. Khoury, B.A. Ovrut, P.J. Steinhardt and N. Turok, Phys. Rev.D 66, 046005 (2002).

[35] L.V. Joukovskaya, Ya.I. Volovich, arXiv:math-ph/0308034.

[36] N. Moeller and B. Zwiebach, J. High Energy Phys. 10 (2002) 034.

[37] Ya.I. Volovich, J. Phys. A 36, 8685 (2003).

[38] L. Brekke, P.G. Freund, M. Olson and E. Witten, Nucl. Phys. B302, 365 (1988).

[39] S. Yu. Vernov, astro-ph/0612487.

[40] Ya. Volovich, Licentiate thesis, Vaxjo Universitet, 2006.

[41] V.S. Vladimirov, arXiv:math-ph/0507018; Theor. Math. Phys. (Engl. Transl.) 149, 1604 (2006).

[42] L.V. Joukovskaya, Theor. Math. Phys. (Engl. Transl.) 146, 335 (2006), arXiv:0708.0642.

[43] V.S. Vladimirov and Ya.I. Volovich, Theor. Math. Phys. (Engl. Transl.) 138, 297 (2004).

[44] A. Sen, Int.J.Mod.Phys. A20, 5513 (2005).

[45] M. Gasperini and G. Veneziano, Astropart. Phys. 1, 317 (1993); M. Gasperini and G. Veneziano, Phys. Rept. 373, 1 (2003); J.E. Lidsey, D. Wands, E.J. Copeland, Phys. Rept. 337, 343 (2000).

[46] T. Biswas, A. Mazumdar, W. Siegel, J. Cosmol. Astropart. Phys. 03 (2006) 009; T. Biswas, R. H. Brandenberger, A. Mazumdar, W. Siegel, arXiv: hep-th/0610274.

[47] V.A. Rubakov, Theor.Math.Phys. (Engl. Transl.), 149, 1651 (2006).

[48] R. Gannouji, D. Polarski, A. Ranquet, A.A. Starobinsky, J. Cosmol. Astropart. Phys. 09 (2006) 016.

[49] C. Armendariz-Picon, V. Mukhanov and P.J. Steinhardt, Phys. Lett. B 85, 4428 (2000); C. Armendariz-Picon, V. Mukhanov and P.J. Steinhardt, Phys. Rev. D 63, 103510 (2001); A. Vikman, Phys. Rev. D 71, 023515 (2005). 
[50] A.A. Andrianov, F. Cannata and A.Yu. Kamenshchik, Phys. Rev. D 72, 043531 (2005); J. Phys. A 39, 9975 (2006).

[51] S. Capozziello, S. Nojiri and S.D. Odintsov, Phys. Lett. B 632, 597 (2006); Sh. Nojiri, S.D. Odintsov and Sh. Tsujikawa, Phys. Rev. D 71, 063004 (2005).

[52] B. Feng, M. Li, Y.-S. Piao, X. Zhang, Phys. Lett. B 634, 101 (2006).

[53] H. Wei and R.-G. Cai, Phys. Lett. B 634, 9 (2006).

[54] D.V. Prokhorenko, arXiv:math-ph/0611068. 